\title{
Social Behavioural Change Communication (SBCC) Strategies for Community Awareness, Mobilization and Participation for Adoption of Arsenic Free Water Consumption Practices -Pakistan
}

\author{
Islam-ul-Haque ${ }^{1}$, Munir Khan ${ }^{2}$, Muhammad Shafqat ${ }^{3}$, Shahid Durez ${ }^{4}$ \\ ${ }^{1}$ Chairman Ecological Sustainability through Environmental Protection Services, Islamabad, Pakistan \\ ${ }^{2}$ Human Resource Development Society (HRDS), Islamabad, Pakistan \\ ${ }^{3}$ Human Resource Development Society (HRDS), Islamabad, Pakistan \\ ${ }^{4}$ Water and sanitation agency, Rawalpindi, Pakistan
}

\section{Email address:}

islamhaq3@yahoo.com (Islam-ul-Haque),munir@hrdsociety.org.pk (M. Ahmed), shafqat@hrdsociety.org.pk (M. Shafqat), shahiddurez1@yahoo.com (S. Durez)

\section{To cite this article:}

Islam-ul-Haque, Munir Khan, Muhammad Shafqat, Shahid Durez. Social Behavioural Change Communication (SBCC) Strategies for Community Awareness, Mobilization and Participation for Adoption of Arsenic Free Water Consumption Practices -Pakistan. International Journal of Environmental Monitoring and Analysis. Special Issue: Ground Water Arsenic Contamination and Action Plan for Mitigation. Vol. 3, No. 3-1, 2015, pp. 56-66. doi: 10.11648/j.ijema.s.2015030301.17

\begin{abstract}
Arsenic contamination has emerged as a serious public health concern in Pakistan. Arsenic is an emerging serious issue at least in two provinces Punjab and Sindh where about 3\% and 16\% of water sources are contaminated with levels of arsenic over $50 \mathrm{ppb}$. The percentage of water sources with concentrations above the WHO level of $10 \mathrm{ppb}$ is $20 \%$ and $36 \%$ respectively in Punjab and Sindh. Both shallow and deep sources have arsenic contamination and therefore testing of every water sources is necessary. A recent study on prevalence of arsenicosis confirmed presence of 40 cases in the study population giving a prevalence 140/100,00 for established and borderline cases. Keeping in mind its adverse impacts on human health, government with financial support of UNICEF launched various arsenic mitigation programmes and installations of various arsenic removal technologies, i,e household levels water filters, community based arsenic removal tank units and deep boring interventions. Unfortunately, all these interventions proved to be un-sustainable primarily due to devoid of community ownership. Lack of awareness, illiteracy and unfavorable socio-economic conditions make the end users in villages/rural areas, the most vulnerable to the adverse effects of any type of contaminated water. The overall increase in illiteracy rates in Pakistan, particularly in rural environment are the contributing factors which magnifies the mind set of arsenic affected communities to adopt safe drinking water best practices. Hence, there is a dire need to develop a sustainable and effective mechanism for Social \& Behavioral Change Communication (SBCC), including the development of communication support materials, within the government structure such as health, education, and social welfare, with the support from print \& electronic media and civil society. Though lot of efforts were made yet the current level of awareness cannot be regarded as satisfactory. Thus keeping view the importance of behavioral change communications, comprehensive communication behavioral change strategies, based on Knowledge, Attitude \& Practices ( KAP) were evolved and implemented in the arsenic hit areas to ensure community mobilization, participation and ownership which is an important aspect towards post arsenic mitigation projects sustainability.
\end{abstract}

Keywords: Behavioral Change Communication, Arsenic Effected Communities, Electronic and Print Media

\section{Introduction}

Pakistan, following the arsenic crisis in Bangladesh and other neighboring countries, has recognized the need of assessing drinking water quality for arsenic contamination. In this regard, the Government of Pakistan has been undertaking many initiatives with assistance from UNICEF since 1999. As a result of these initiatives, the presence of arsenic 
contamination has been recognized and consequently an arsenic mitigation programme launched by the government of Pakistan with the technical \& financial assistance of UNICEF. During this process experiences have been gained related to planning, implementation, monitoring and management of arsenic mitigation(Bilal et al, 2008).

Based on the findings of the preliminary investigations in 2000, UNICEF extended technical and financial support to the Public Health Engineering Department (PHED) and Local Government and Rural Development (LG\&RD) to launch an intensive and extensive National Survey (2001) to further assess the level of arsenic contamination beyond the six districts considered during the preliminary investigation. This time one-third of all the districts in the country (i.e., 35 out of 104 districts) were selected from all the four provinces of Pakistan. Keeping in view the adverse impacts of arsenic contamination on human health, government pf Pakistan with the financial support of UNICEF \& other donor agencies launched series of arsenic mitigation interventions , mentioned as under(Islam, 2015);

a. Installation of community based arsenic removal technology units.

b. Provisioning of deep boring / bore for arsenic free water

c. House hold levels arsenic removal filters and clay pitchers

But un-fortunately, all these interventions became unsustainable due to devoid of community participation and ownership. Lack of awareness, illiteracy and unfavorable socio-economic conditions make the end users in villages/rural areas, the most vulnerable to the adverse effects arsenic or of any other type of contaminated water. Hence, there is a dire need to develop a sustainable and effective mechanism for Social \& Behavioral Change Communication (SBCC) strategies for changing the mind sets of affected communities for adoption of safe drinking water practices,

The initiative employs an integrated strategy comprising intensive stakeholder participation, including the local communities, social mobilization, capacity building, awareness, and culturally appropriate behavior change communication interventions that are in line with proposed KAP (Knowledge, Attitudes, and Practices) guidelines and strategies. It will provide support to Tehsil Municipal Administration (TMA)/Local Government \& Rural Development Department (LG \& RDD), Public Health Engineering Department (PHED), UNICEF, WHO, Pakistan Council of Research for Water Resources (PCRWR) and non government organizations (NGOs), community based organizations (CBOs) (HRDS Report, 2014) and communities through capacity building and training in operations and management of water resources \& WASH facilities, hygiene and sanitation promotions, community mobilization, cost recovery, Planning, Finance and Monitoring and Resource mobilization; Participatory Planning and monitoring skills . Public \& private partnership, Community \& Leadership Skill Management Training (CMST/LMST), water sampling, testing and disinfecting; sanitary inspection. The Community
Resource Persons ( CRPs) / activist were indentified and were tasked community triggering for their mobilization, participation and ownership, based on cascading model, as shown in figure-1, below;

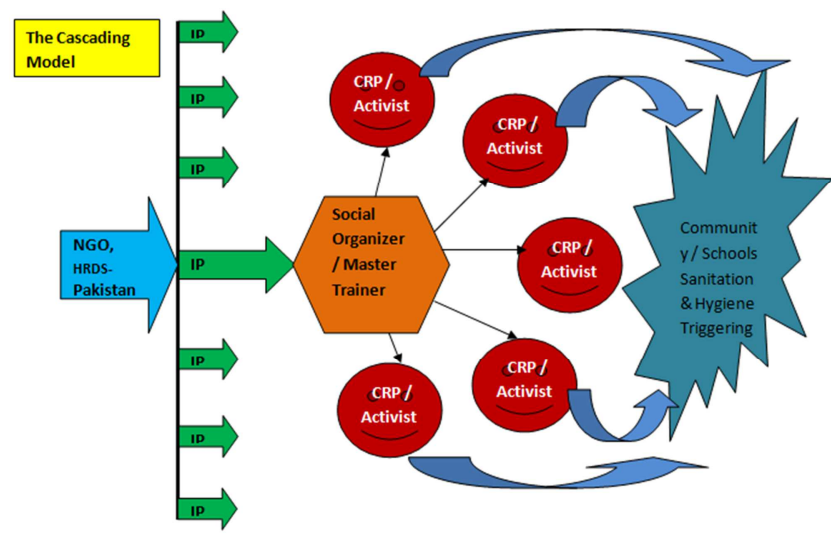

Figure 1. The cascading community triggering model for effective community mobilization, participation and ownership, carried out by HRDS with its implementing partners (IPs) in arsenic hit areas of Pakistan.

A comprehensive SBCC strategy has specifically been designed to improve the health status of people in the country regarding adopting arsenic free safe drinking water . Diverse awareness and SBCC activities will seek to increase awareness and knowledge at the grassroots level aiming at bringing positive and lasting changes in Knowledge, Attitudes, and Practices at the household level, as well as NGOs / CBOs etc . Local NGOs/CBOs will monitor them and report on their activities apart from providing them backstopping support. Besides tapping local events other diverse community based awareness and BCC activities such as interactive theaters, festivals for safe water \& hygiene promotion. Activities/events will reinforce inputs into the awareness and SBCC interventions. It will also provide a comprehensive review of water treatment and water quality testing technologies for use at the community and household levels. The strategy entails water testing and promotion of Household water purification technologies. Local NGOs/CBOs will incorporate follow-up of these activities in their operations in affected areas and will try to consolidate the SBCC campaign which is main stay of lasting sustainability any programme.

\section{Literature Review}

A helpful framework of potentially influential factors impacting the use of safe drinking water options is the Health Action Process Approach (HAPA; [Schwarzer, 2008]). This theory has been successfully employed for explaining many health behaviors [Schwarzer, 2008]. The HAPA specifies risk perception, outcome expectancies, selfefficacy, and planning as behavior-influencing factors. The success of any programme or project hinges around the levels of community participation and ownership which is only possible by designing and implementing effective social behavioral change communication strategies . Besides 
the psychological factors related to the target behavior, it is argued that it is important to consider the social-cognitive factors for regarding the competing behavior. Every behavior has an alternative, and new behaviors are weighed against the old behaviors that serve similar purposes. Therefore, world over, the importance SBCC has been recognized as main stay of successful programmes

To avoid most health threats, including arsenicosis, it is necessary not only to adopt a healthy behavior but also to maintain this practice over time. Therefore, the goal of every behavior change campaign is to induce long-lasting behavior change. One indicator of sustained behavior is habit. Habits facilitate intended behaviors because they require reduced cognitive effort [Tobias, 1998]. Therefore, as also suggested by Verplanken and Wood [Verplanken et al, 2006], we propose that developing habits should be considered an important additional goal of behavioral change campaigns. Consequently, a habit should be considered a dependent variable in statistical investigations to enable the identification of its determinants, which can be targeted in behavioral change campaigns cf. [Orbell et al, 2010, Verplanken et al, 2008]. However, explaining and predicting habits alone does not seem sufficient. In the case of promoting new behaviors, some individuals may already be exhibiting these new behaviors, but they may not have developed into habits yet. However, other individuals may not be exhibiting the behavior yet. To consider all individuals simultaneously, the actual behavior and the stability of this behavior should be considered (i.e., the habit). In this study, we use the concept of habitual behavior, which considers both the habit and the actual behavior.

\section{Objectives}

The salient objectives of social behavioral change communication strategies are as under;

a. To identify and discuss various approaches for social behavioral change communication strategies suitable for high risk arsenic contamination affected areas and communities.

b. To design and formulate social behavioral change communication strategies for arsenic affected communities Pakistan for long lasting and sustainable arsenic mitigation programmes.

c. To discuss and evaluate the impacts of social behavioral change communication strategies( SBBC)

\section{Methodologies}

Knowledge, Attitude \& Practices ( KAP) survey was conducted in the arsenic contamination hit / affected areas to ascertain the socio-economic, literacy rate, water storage $\&$ treatment practices, average incomes and gender composition etc. Few of the findings of KAP, conducted in district Muzaffargarh, are shown in tables-1, 2 \& 3, below ;
Table 1. Percentage of Households Using Various Methods to Purify Water.

\begin{tabular}{ll}
\hline Ways to purify drinking water & Percentage \\
\hline None & 90.0 \\
Boil & 8.8 \\
Use chlorine tablet & .8 \\
Filter with clothe and sand & .8 \\
Total & 100.0 \\
\hline
\end{tabular}

Table 2. Percentage of disease occurrence in the last two months according to gender.

\begin{tabular}{lll}
\hline Disease & Female & Male \\
\hline Diarrhea & 48.8 & 36.8 \\
Typhoid & 16.9 & 11.9 \\
Dysentery & 1.3 & 1.3 \\
Jaundice & .6 & 3.8 \\
Skin disease & 28.8 & 31.3 \\
Malaria & 18.8 & 21.9 \\
\hline
\end{tabular}

Table 3. Electronic Media Sources Ownership.

\begin{tabular}{ll}
\hline Media Source & Percentage \\
\hline Television & 11.3 \\
Radio & 8.1 \\
DVD/VCR & - \\
\hline
\end{tabular}

\subsection{Communication Approaches and Methods}

Thus basing on the findings of the KAP survey, the following communication approaches were employed in designing the SBCC activities within the affected areas to determine the most effective medium for intervention in order to adapt the strategy. Positive deviance - an "assetsbased" behavior change approach, which improves outcomes by capitalizing on existing community strengths .

\subsubsection{Interpersonal Communication (IPC)}

IPC proved to be the key medium for SBCC interventions in affected areas which includes workshops, meetings, seminars and community awareness sessions .

\subsubsection{Mass Media to Support IPC Activities in the Affected Areas}

Mass media was used to raise awareness and advocate for adoption of arsenic free/safe water management, hygiene and sanitation practices. Mass Media ( mobile phone messaging, television, print, and FM radio) were utilized to disseminate health and hygiene messages, both written and pictorial, and provide information on numerous safe practices. This included radio spots, print articles, public service announcements, and aired focus group discussions.

\subsubsection{Alternative Media}

Possibilities to develop audio drama dialogues such as conversations between a mother-in-law and a daughter-in- 
law wherein the former is heard to be providing sound and reasonable advice to the latter pertaining to arsenic free / safe health hygiene and sanitation practices were explored. This was played during women's gatherings or if appropriate on the radio to spread mass awareness.

\subsubsection{Information, Education and Communication: (IEC)}

IEC material was used during IPC, mass media, stakeholder workshops and during capacity building trainings. Hygiene and sanitation messaging will be very specific and targeted to each of the hygiene and sanitation interventions, including drinking arsenic free water. For example, female Community Development Officers (CDOs) meet and speak with women in their villages to discuss hygiene and sanitation issues. Messaging campaigns include messages regarding the arsenic contamination water testing and purification technologies, symptoms, prevention methods . Community was educated to use the water from safe sources i.e water source marked with paint green for arsenic safe sources and not the red for sources with $>50 \mathrm{ppb}$ Arsenic. These messages were disseminated widely, including during community events. These messages were also made part of school-based activities which magnified their outcomes and impacts .

\subsubsection{Stakeholder Participation}

A consultative meetings was held during the planning stages to provide an opportunity to various stakeholders to share their experiences on arsenic free drinking water practices with improved hygiene and sanitation SBCC strategies. The SBBC strategy engaged the following stakeholders;

a. Tehsil Municipal Administration (TMA)/local governments.

b. Public Health Engineering Department (PHED) who are responsible for the provisioning of safe water

c. Non government organizations (NGOs), community based organizations (CBOs) etc.

For follow-up meetings on a regular basis to share progress, obtain feedback, address emerging issues, and ensure ongoing support, District Health Hygiene Management Teams (DHHMTs), and Quality Improvement Teams (QITs) were constituted from these diverse set of public, NGOs/CBOs, and private sector representatives.

The DHHMTs and QITS were formed for proper management and technical quality improvement of safe water management, health and hygiene promotion within the affected districts. The idea of DHHMTs and QITs was to prepare the stakeholders for an ongoing management and progressive improvement of arsenic free / safe water management and health and hygiene promotion. The stakeholder will also see possibility for advocacy on the following:

- Conduct Blanket survey for arsenic contamination in affected districts of Punjab \& Sindh whereas random/selective sampling in suspected districts.
- Identify the high risk areas for arsenic contamination.

- Mobilize and prepare the community for use of Arsenic mitigation techniques in the identified arsenic affected areas

- Introduce low cost arsenic removal filters in affected areas.

- Deep boring water supplies (alternative safe water sources)

- House hold arsenic removal filters

- Chlorine tablets for bacterial contamination

These initiatives assumed that all these stakeholders will be acting in tandem for safe water management and hygiene promotion activities. This involvement from project design to participatory monitoring and evaluation will develop ownership to ensure sustainability of SBCC interventions.

Before the initiation of field activities relevant officials responsible for the district were be approached for further necessary administrative permissions and consultations.

\section{Results and Discussion}

\subsection{Community Mobilization}

A fundamental behavior change principle is promoting social changes amongst the target communities. In order to promote social changes, community engagement, efficacy, and empowerment are the central to sustain new behaviors. Community mobilization activities were carried out primarily at the village level, based on the premise of communities themselves identifying problems and goals, mobilizing resources and developing and implementing strategies to address their community health problems. Towards this end, Community Development Officers (CDOs) indentified and/or form, strengthen, sensitize and mobilize CBOs on arsenic free/safe drinking water management, health and hygiene promotion. Water User Committees (WUCs) were constituted of suitable activists from the CBOs. The CBOs/WUCS provided institutional capacity through Community \& leadership Management (C \& LMST) skills trainings. Along with the public sector entities such as the local government, TMA, and PHED CBOs/WUCs representatives were also capacitated in operations and management of arsenic/safe drinking water resources, hygiene and sanitation promotion, community mobilization, cost recovery, Planning, Finance and Monitoring and Resource mobilization, Participatory Planning and monitoring skills, Public \& private partnership, Community \& leadership Skills Management Training (C \& LMST), water sampling, testing and disinfecting; sanitary inspection. This all round capacity building of CBOs has been designed to enable them for self sustaining sensitization of key audiences such influential community members/religious elders, prayer leaders, male family members, mothers, school teachers etc. With CBOs strengthening on these lines SBCC managers expect that they will continue their Hygiene promotion activities even without significant external backstopping. 
The community mobilization triggering process flow mechanisms is shown in figure-2, below;

\section{Community Mobilization Triggering Process Flow Mechanisms}

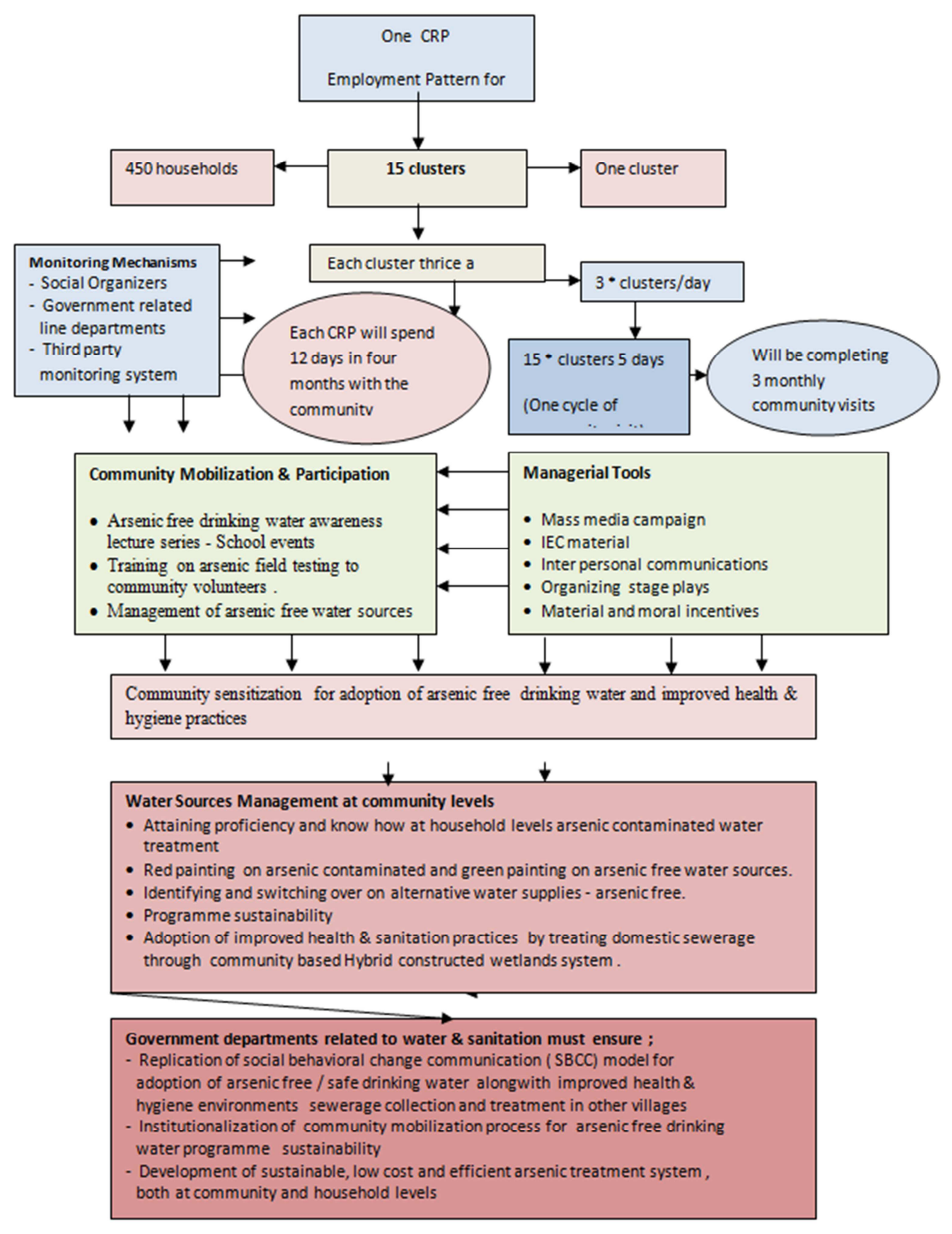

Figure 2. Community Mobilization Triggering Process Flow Mechanisms for community participation.

Various arsenic mitigation activities are being performed by the community after community triggering sessions which is quite enough for programme sustainability. The arsenic testing activities by the community are shown in figure-3, below;

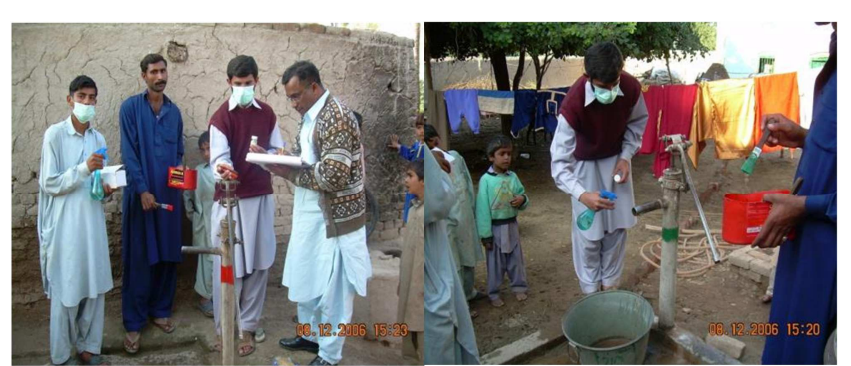

Figure 3. Community volunteers are marking arsenic contaminated water sources with red paint and also taking water samples for arsenic testingarsenic contaminated village-Pakistan. 


\subsection{Core SBCC Program Activities}

Based on information regarding maternal and caretaker knowledge, attitude, and practices collected through KAP assessments in each area and using project material implementers undertook, following SBCC strategies for core arsenic free drinking water \& hygiene and sanitation promotion activities which created adequate awareness, ownership and sustainability to arsenic mitigation programmes;

\subsubsection{Stakeholder Capacity Building}

In order to operate on a self-sustained basis in their respective communities, local NGOs / CBOs, doctors , health service providers, teachers, community members, parents and women/mothers, prayer leaders, trained on project materials and Hygiene Promotion (HP) strategies by a team of Master Trainers (MT), both male and a female, assisted by Community Development Officers (CDOs), both male and female. A separate programme familiarization session was arranged for print \& electronic media and journalists for wide spread programme promotional activities.

Community awareness sessions were organized at the grassroots level for these audiences. They will be trained in communication, group facilitation skills, and use of Information, Education and Communication (IEC) materials especially within the intervention packages for arsenic free water and improved health, hygiene and sanitation education to spread health messages at the community level. They organized community awareness sessions /activities on numerous hygiene and sanitation topics for their respective target audiences. Local doctors and health workers in health facilities, but also with private providers, including Lady health Workers(LHWs), Traditional Birth Attendants (TBAs) were trained in the appropriate use of IEC and hygiene and sanitation messaging to increase their own knowledge about safe water, hygiene and sanitation practices. This helped them to disseminate the messages to the communities with whom they work affectively. The few glimpses of capacity building activities are shown in figure-4, below;

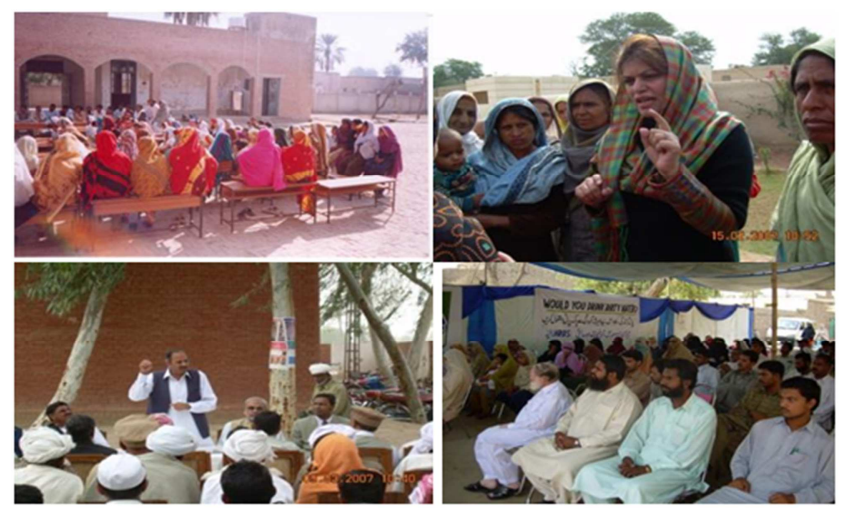

Figure 4. Community Awareness and capacity building sessions in arsenic affected areas for arsenic mitigation programme ownership and sustainability.
These trained segments of communities were also sensitized and mobilized to operate on a self-sustained basis in their respective communities, helping parents, women and children at their homes. As they come from the local community which made it easier for them to reach their clients. Local NGOs/CBOs monitored them periodically according to an appropriate format meeting the requirement of monitoring information. Quarterly reports were obtained on their activities. These trainings are expected to ensure standardization of knowledge throughout the affected districts leading to better program implementation and participatory monitoring of SBCC activities.

The scope of training activities was as under ;

- Staff capacity building and training to implement the BCC strategy in close coordination with District Administration, Local Government/TMA, PHED staffs, Medical Superintendent / Deputy Medical Superintendent (MS/DMS) District head quarter Hospital (DHQ) Hospital, and CBOs/NGOS etc.

- Development \& finalization of IEC material.

- Undertaking HP sessions with women (Mothers and Traditional Birth Attendances-TBAs)

- Undertaking HP sessions with men/village

- Dissemination of IEC material in routine activities.

- Distribution of Hygiene Promotion kits (Soap, Comb, Towel, MISWAKs, Nail clippers) at house hold (HH) level. This will serve as incentives.

- Identification, training and support to women and youth activists / volunteers as Hygiene promoter.

- Training programs for local NGOs/CBOs/WUCs for HP messages integration in their routine activities; (12-15 participants/Training)

- Trainings for Paramedic staff of District \& Tehsil Head Quarter Hospital, BHUs and private practitioners on use of project materials and HP strategy (12-15 participants/Training)

- Trainings for Local Government \& Rural Development Department (LG \& RDD)/Tehsil Municipal Administration (TMA), PHED staff and local elites on HP measures (12-15 participants/Training)

- Trainings for Imams and religious leaders on use of project materials and HP strategy (12-15 participants/Training)

- Identification and 5 days lead training of at least 2 volunteers in each village for interactive performances

- Organizing interactive theaters every 6 months.

- Organizing $1 \mathrm{Mela} /$ festival/tehsil/6 months.

- Organizing HP activities tapping local events: 2 event/tehsil/6 months.

- Organizing HP activities including water testing and promotion of water household water purification technologies at House Hold Levels at Mohalla, Village, Union Council, and Tehsil.

- Water quality testing (100 samples/tehsil/12 months)

- Establishment of linkages with local media (cable, radio, newspaper) to promote HP messages

- Arrange and organize quarterly review meetings at 
District levels, participated by local administration, Local Government \& Rural Development Department (LG \& RDD) and all other stakeholders .

The related government staff, community activists and volunteers amongst the community were trained on water testing and water quality aspects. The knowledge gained from this training was further imparted to affected areas communities by this trained staff. Various training activities are shown in the figure-5, below;

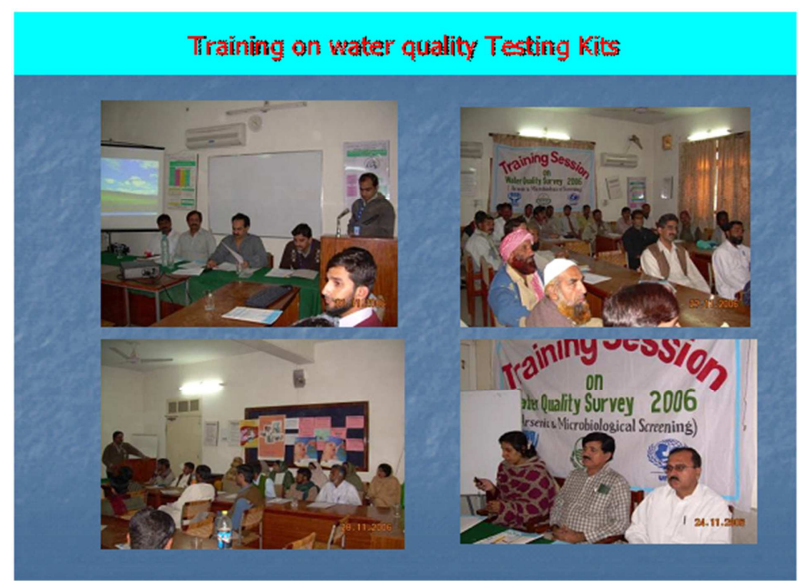

Figure 5. Various training activities for government department staff and other stakeholders.

The village levels theaters proved to be very helpful in creating awareness regarding ill-impacts of drinking arsenic contaminated water on human health. The theater activities are shown in figure-6, below;
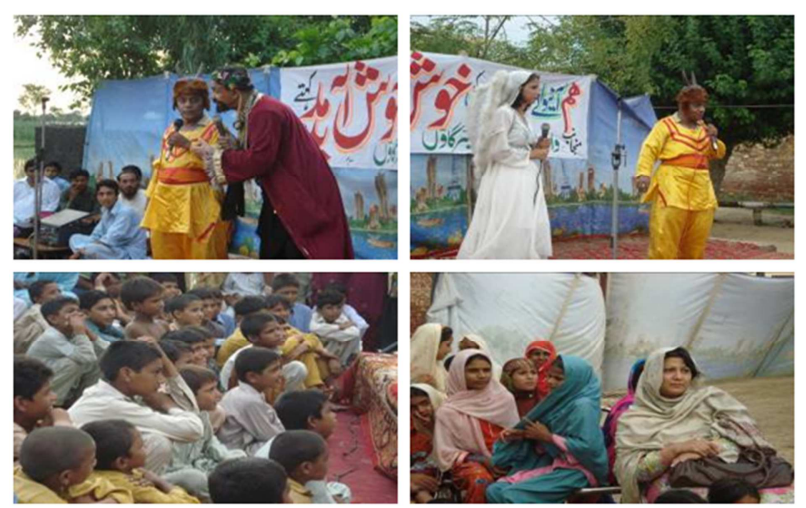

Figure 6. Street theater activities for school children and communities in arsenic contamination affected areas for creating awareness regarding arsenic mitigation programmes.

\subsubsection{School Component}

In order to operate on self-sustained basis in their respective communities during the course of the project and beyond, using 1-day curricula, Master Trainers and Monitors trained teachers in groups of 25 - one teacher per primary school - under school component of the strategy. They were be trained in communication, group facilitation skills, and use of Information, Education and Communication (IEC) materials especially within the intervention packages for arsenic free drinking water $\&$ health, hygiene and sanitation education to spread health messages at the school level. Awareness behavior change activities were conducted by the teachers on regular basis at the schools for children of class 4 within the intervention packages for health, hygiene and sanitation education. Those trained were also sensitized and mobilized to operate on a self-sustained basis in their schools helping children in key hygiene and sanitation practices. A mechanism to monitor them periodically according to an appropriate format meeting the requirement for monitoring information was also devised.

The school component has been designed on the premise that children are not only adversely affected due to poor water management, hygiene, and sanitation they also have the potential to disseminate the messages to peers, siblings, and parents. While the trained teachers expressly sensitized and mobilized to encourage the children for wide dissemination especially among the peers, siblings, and parents.

The full scope of activities was as under;

- Train, using 1-day curricula, groups of primary school teachers in groups of 25 (one teacher per primary school) in School safe water and Hygiene Activities.

- Facilitate one trainer from the office of EDO, Education to attend each training event.

- Monitor \& Report activities at all primary schools where teachers are trained once in a quarter.

- Conduct pre and post tests $4^{\text {th }}$ class students in a given number of schools in affected tehsils each year according to carefully developed protocol.

Interactive sessions on creating awareness on drinking arsenic free water and improved health \& hygiene session with school children is shown in figure-7, below;
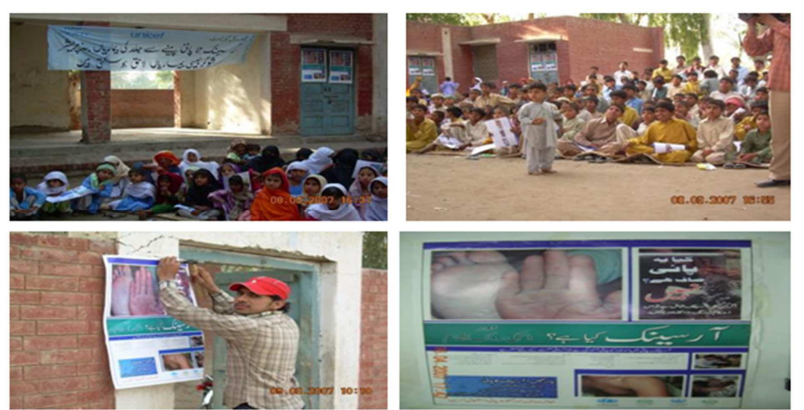

Figure 7. Various interactive sessions with school children, both male \& female and display of arsenic contamination awareness posters in school premises.

\subsubsection{Capacity Building of the Local Communities}

The intervention aimed at forming a committee of beneficiary households or strengthen and mobilize an existing $\mathrm{CBO} / \mathrm{CO}$ to take over the responsibility for operation and maintenance of the WASH facilities, including use SBCC strategies to bring the behavioral change amongst affected people for adoption of arsenic free drinking water practices of in partnership with the local government .

Under the capacity building component a team of Master Trainers (MT), both male and female, assisted by Community Development Officers (CDOs) both, male and a 
female was established to enhance the capacity of Water Users Committees (WUCs) in the affected districts. The WUCs were selected from the CBOs. This intervention ensured the success, desired output, and sustainability of similar initiatives and ensure the sustainability of outcomes. The established WUCs was responsible for the existing and upcoming safe water supply and sanitation and other schemes by taking over their O\&M responsibilities. A communitybased approach involving people at the grassroots level to support these initiatives was employed which proved to be very effective for arsenic mitigation programme sustainability and ownership.

\subsection{District Health and Hygiene Management Team (DHHMT)}

A District Health \& Hygiene Management (DHHMT) team was constituted comprising members such as Deputy Commissioner, Assistant Commissioner, Local Government/TMA and PHED officials, Medical Superintendent (MS), Deputy Medical Superintendent DMS DHQ \& THQ hospitals, related public and private sector health professionals, representative of PPHI, UNICEF, WHO, community members who have some interface with the issue, local NGOs/CBOs involved in safe water management, health and hygiene;

All these members were encouraged to draft a medium to long term plan for safe water management, health and hygiene promotion at the district level, its goal, objectives, and the activities. Work plans, progress, and important information regarding all activities on safe water management, health and hygiene promotion at the district level were shared with them whereas besides acting as a team they will meet regularly to discuss the smooth progress of the activities and coordinate their actions to support the objectives of the safe water management, health and hygiene promotion at the district level.

Ideally the DHHMT should hold regular meetings to coordinate the activities of the numerous departments aimed at maximizing the benefit to the people of the area. It is expected that regular sharing of information and coordination of activities will remove problems in efficient delivery of safe water management, health and hygiene promotion services in the district. Besides helping overcome challenges of the safe water management, health and hygiene promotion in the district this team will also help attend myriad issues such as lack of doctors, health workers, medicines, vaccinations, equipment, infrastructure, and security etc.

\subsection{Quality Improvement Teams (QITs)}

Quality Improvement Teams (QITs) were constituted of persons with diverse backgrounds so that the knowledge, skills, experience and perspectives of different individuals are harnessed to make lasting improvements in technical, managerial, social aspects of the services for safe water management, health and hygiene promotion. It included Health Ministry, Health Secretariats/Directorates, UNICEF,
WHO, professionals, health workers, nurse, public sector concerned officials, and community activists with related backgrounds.

This team made concerted efforts on a continuous basis to find better ways to provide better care and service to children and women in terms of safe water management, health and hygiene promotion. They considered the process or the system in its numerous aspects. People with diverse backgrounds but having interface with health practice will be able to consider every dimensions of safe water management, health and hygiene promotion. They worked diligently and focused on identifying the key problems in actual practice and pondered over solutions. They regularly collected data, processed and analyzed it, made recommendations and took appropriate actions.

\subsection{Medium to Long Term Sustainability}

The strategy has been designed with a view to ensure the sustainability of the inputs. Community Based Organizations and their formation, training and sensitization is designed to anchor the initiatives in the community and create local capacity and demand. All stakeholders ensured viability of the CBOs through ensuring their regular backstopping and this possibly helped their establishment on sound footings.

Sensitization and mobilization i.e sessions and numerous activities with influencers such as maliks, elders, \& religious leaders addressed challenges posed by the belief and practices entrenched on the basis of culture, custom, norms, and religion. The Communities were orientated \& sensitized on safe water management, health and hygiene promotion interventions which included parents, teachers, community members, maliks, elders, and religious leaders. The beneficiaries were fully made aware of best practices on sanitation, clean water, personal \& environmental hygiene, arsenicosis and diarrhea prevention, danger signs of arsenicosis \& diarrhea, diseases . Through school-based activities and IEC distribution this process was further strengthen and reinforced.

Apart from women and men activists especially trained for dissemination of messages sensitization work with most of stakeholders has been designed to train them for wide dissemination of the knowledge on a self sustaining basis. Community health workers trained, will also operate on a self-sustained basis in their respective communities, helping mothers and children at their homes. They will be residents of the communities in which they work, and work out of their homes, which will make it easier for them to reach their clients. Through the LHWS \& TBA the system for referral will be also improved.

It is expected that the methodology adopted will enable the sustainability of this knowledge and will lead to sustainable best practice.

\subsection{Performance Management Strategy}

A detailed monitoring and evaluation plan was developed 
by the key stakeholders. Monthly meetings were coordinated and held to discuss the progress of activities and to identify any corrective actions that needed to be taken. Once the baseline data for the indicators are available, they should be placed in an indicator matrix detailing numerator, denominator, baseline value, target values, and details on collection (frequency, person responsible, etc). Routine monitoring included visits by activity staff to target communities to assess program implementation, program records on trainings conducted, and reports by trainers. The CBOs served as a feedback mechanism to ensure that various meetings, sessions, and events are taking place according to the implementation plan.

\subsection{Monitoring, Tracking and Evaluation: Indicator and Means of Verification}

Indicators and means of verification should be outlined in detail. Key monitoring indicators will be No. of stakeholders trained viz. by type, by gender; no. of activities conducted viz. by type, by gender; No. of people reached for each type of activity and gender; No of communication material disseminated by organization and type; and no. and type of mass media aired or printed by project. Beyond that stakeholders should identify and track indicators for quality such as the no. of people adopting various prescribed safe practices. Reports prepared for sponsors on a regular basis, field visit notes, research studies, and evaluation reports were also used as a means of verification. The monitoring process by the donors (UNICEF) is shown in figure-8, below;

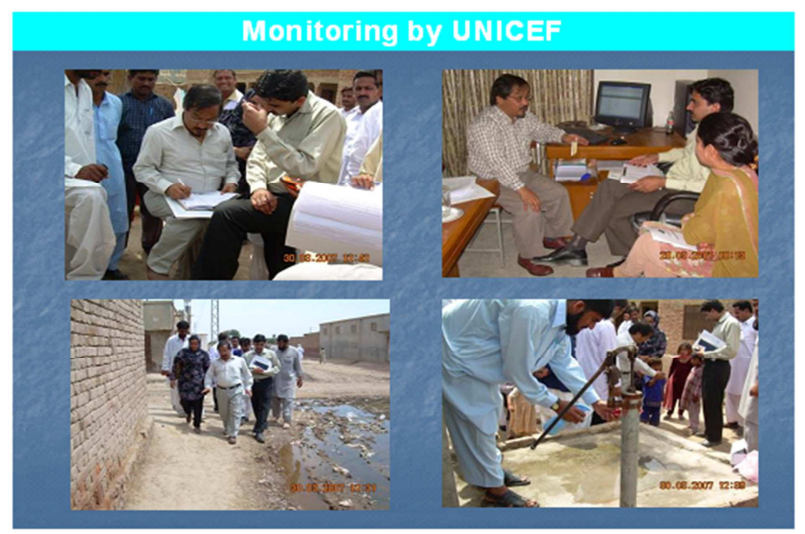

Figure 8. WASH specialist of UNICEF is checking various field activities, being carried out by HRDS in arsenic contaminated areas, under social behavioral change communication implementation programme.

For monitoring and evaluations, following methodologies were adopted.

\subsubsection{Monitoring and Tracking Direct Beneficiaries}

The program monitoring possess was embedded with the following three major components;

\section{(i) Databases and Tracking}

A database was developed for tracking direct project beneficiaries. This database included information about each individual direct project beneficiary (stakeholders / volunteers trained) and was used particularly for tracking stakeholders' activities. Besides program monitoring the analysis generated from information of this database was /can also be utilized for defining project activities, for instance if a particular area is registering a high rate of arsenicosis or diarrhea, then efforts should be focused in that area.

\section{(ii) Indicator Monitoring}

Data was collected on each indicator on a monthly basis and specifically designed tools were used for the purpose. Analysis of this data was carried out on SPSS and with periodic review i.e monthly, quarterly etc. The results were shared with all the concerned stakeholders for taking appropriate action for bring improvements.

\section{(iii) Process Monitoring}

Process monitoring was also carried out at two levels; the staff from sponsors may visit the project sites, monitor the pace and quality of activities, and provide feedback to project staff. Secondly, the monitoring staff, along with, collecting data for indicator monitoring should also be providing feedback and input on the pace of activities. Process monitoring followed the principles of participatory monitoring mechanisms and communities were actively engaged through focus group discussions and meetings to provide a beneficiary perspective. This enabled the project team to respond to any issues arising in the project in a timely manner.

\section{(iv) Evaluation Plan/Mechanisms}

Evaluation was planned to be carried out at two stages / levels, a project evaluation and an activity evaluation, with the project evaluations at the end of the project. External evaluators conducted the evaluation. For activity evaluations, the project monitoring staff conducted studies on some of the key activities of the program i.e. quality of trainings, trainers' effectiveness, and role of community groups in conducting monitoring of activities. These evaluations started around mid of the project and continued till the completion of the project. The recommendations of these studies were shared with the project management team and were incorporated into the program design. The purpose of these evaluation reports were strengthening the monitoring mechanisms for keeping a quality check on the activities and for course correction.

\section{(v) Performance Monitoring Matrix}

The Program performance monitoring matrix outlines the project performance monitoring plan. At the start of the program, quarterly assessments was to be done to examine the effectiveness of SBCC strategy components. Implementers included questions to assess levels of participation of the stakeholders in program activities and other relevant indicators.

\subsection{Risks, Challenges and Constraints}

The heightened security situation in the country is a factor that must be taken into consideration when planning and 
implementing programming. It is important to note that continued violence and general insecurity may affect program implementation. Other constraints include: low literacy levels, particularly among women and challenges in reaching women and children due to socio-culture factors and difficult geographic terrain.

Reliance was made on to the local implementers enjoying a high level of acceptance in the arsenic water contamination hit areas who are cognizant of cultural values, have regular stakeholder contacts, and continuous monitoring of activities, paying close attention to community responses. A security policy and standard operating procedures were worked out and documented to deal with such law \& order situations. Before embarking on program activities an initial security assessment of all sites was carried out / undertaken prior to implementation. This assessment \& monitoring served the foundation for the daily security management plans for operations and security plans was embedded with flexibility of incorporating changes, as and when required by the field staff.

\subsection{Impacts}

Social behavioral change communication (SBCC) strategies were implemented in the arsenic contamination affected areas by Human Resource Development Society (HRDS), a national NGO which possesses high credibility in development sector in Pakistan. The evaluation of the projects outcomes were carried out by a third party, with following outcomes \& impacts;

1. The results of the project showed that interventions had laid the foundation of arsenic free and improved hygiene behavior in target communities.

2. An overall knowledge about relationship of drinking arsenic contaminated water with different diseases has increased significantly i.e. from baseline figure of $58 \%$ to figure of $86 \%$ by the evaluation survey.

3. The schoolteachers have been provided training in health and hygiene practices, and these teachers organized the awareness sessions in the schools with the support of social organizer and community resource persons (CRPs). The session included training of children in adopting arsenic free drinking water and improved health \& hygiene practices. The local school teachers in their interviews to the evaluation team informed that a change was seen in personal hygiene of students, and even their families were influenced with their behaviors. The FGDs with children highlighted the knowledge of children in safe drinking water and improved health \& hygiene practices.

4. The CRPs and Social Organizers played a key role in mobilizing the communities. The use of Participatory Rural Appraisal (PRA) tool in the communities specifically transect walk and triggering sessions informed the communities about the poor safe water $\&$ hygiene conditions of their villages, and what are implications if safe health practices are not adopted. The communities have been organized into village wise Water User Committees (WUCs) and sanitation committees (VSCs) to take lead towards safe drinking water
\& improved hygiene practices. The WUCs \& VSCs, as a group, enabled the communities in linking with local government department but equally to understand the role of health and education department. Thus, the intervention has resulted broadening their vision towards the development of village beyond the safe drinking water and sanitation for all.

5. The Community Resource Persons (CRPs) played a key role in creating awareness about arsenic free drinking water and improved health \& hygiene practices. construction of latrines. Behavioral change is a long term process, and duration of the project was short as an early recovery intervention. A CRP has served an average 350 households and, in the present financial circumstances, the government of Pakistan would be reluctant to hire CRPs for long-term engagement even though these CRPs are pivotal to the success of the program. The program needs to explore the options of using the existing public sector units like community development mobilizers working in two provinces of Pakistan. LHWs working at the community level, school teachers, etc. are the potential sources with the government for long term sustainability for SBCC strategies .

6. The project deployed 131 social organizers and 931 community resource persons (CRPs) against the targets of 126 and 826 respectively. These social organizers and CRPs were provided training in community mobilization with a focus on triggering and health hygiene sessions. The reports showed the achievement of target of 2.3 million people for health and hygiene messages, and triggering to generate the demand for safe drinking water \& improved health and hygiene practices through this network.

7. The survey of the evaluation team revealed that $70 \%$ of the households attended arsenic drinking water $\&$ health and hygiene sessions held in their areas. This does not include visits of LHWs to the households or sessions in the schools or theatre groups. The highest numbers are in Bagh (92\%), Jaffarabad $(82 \%)$ and Thatta $(80 \%)$, but were lowest in Charsadda (25\%) and Hunza Nagar (60\%).

8 . The findings suggest that over four-fifths $(85 \%)$ of the communities store their drinking water in a clean container indicating increased community awareness about the importance of safe drinking water practices.

\section{Conclusions}

Ground water arsenic contamination problem is growing in scope and complexity all over the world, particularly in developing countries. Numerous interventions have been taken and are being taken for mitigation of arsenic contamination, mostly by the donor agencies like UNICEF, in Pakistan. Experiences show that all such arsenic mitigation programme could not become sustainable programmes. The devoid of community mobilization, participation and ownership was found to be the salient causes of unsustainability for arsenic mitigation programmes. Government buying and ownership was an other cause of programmes un-sustainability in Pakistan. Therefore, keeping in view the root causes arsenic mitigation programmes un- 
sustainability, a comprehensive social behavioral change communication (SBCC) strategies designed, based on KAP survey findings which helped in designing the area, cultural and socio-economic specific communication material. SBCC strategies were implemented in arsenic hit areas of Pakistan. The programme monitoring and evaluation was conducted by the third party who confirmed dire necessity of SBCC programmes which ensure community mobilization, participation and ownership. Furthermore, under MDG goal 7, Pakistan is obligated to enhance access to safe drinking water and improved sanitation which indicates that more work on provisioning of arsenic free drinking water has to be carried out in Pakistan . SBCC strategies must be planned made mandatory alongwith undertaking any arsenic contamination mitigation projects.

\section{References}

[1] Bilal Akbar, Shafqat Ali, Arsenic Monitoring and Mitigation in District Rahim Yar Khan - Punjab, Progress and Prospects on Water: For a Clean and Healthy World with Special Focus on Sanitation, World Water Week in Stockholm August 17-23, 2008, Page 140-141, Published by Stockholm International Water Institute, SIWI Drottninggatan 33 SE-111 51 Stockholm Sweden

[2] Brewer NT, Chapman GB, Gibbons FX, McCaul KD, Weinstein ND: Meta-analysis of the relationship between risk perception and health behavior: the example of vaccination. Health Psychology, Vol 26(2), Mar 2007, 136145. http://dx.doi.org/10.1037/0278-6133.26.2.136.

[3] Floyd DL, Prentice-Dunn S, Rogers RW: A meta-analysis of research on protection motivation theory. Journal of Applied Social Psychology Volume 30, Issue 2, pages 407-429, February 2000.

[4] HRDS (HEALTH \& RURAL DEVELOPMENT SERVICES) Annual Report 2014, Website: www.hrdsfoundation.org.pk Email: info@hrdsfoundation.org.pk
[5] Islam ul Haque, Performance Assessment of Installed Arsenic Removal Technologies and Development of Protocol for Alternative Safe Drinking Water Supply Options for Arsenic Hit Areas of Pakistan, International Journal of Environmental Monitoring and Analysis 2015; 3(3-1): 31-42 Published online March 4, 2015 (http://www.sciencepublishinggroup.com/j/ijema) doi: 10.11648/j.ijema.s.2015030301.14 ISSN: 2328-7659 (Print); ISSN: 2328-7667 (Online).

[6] Mosler H-J: A systematic approach to behavior change interventions for the water and sanitation sector in developing countries: a conceptual model, a review, and a guideline. International Journal of Environmental Health Research Volume 22, Issue 5, 2012.

[7] Orbell S, Verplanken B: The automatic component of habit in health behavior: habit as cue-contingent automaticity. Health Psychology, Vol 29(4), Jul 2010, 374-383. http://dx.doi.org/10.1037/a0019596.

[8] Schwarzer R: Modeling health behavior change: how to predict and modify the adoption and maintenance of health behaviors. Applied Psychology Volume 57, Issue 1, pages 129, January 2008.

[9] Trafimow D, Sheeran P: Some tests of the distinction between cognitive and affective beliefs. Journal of Experimental Social Psychology, Volume 34, Issue 4, July 1998, Pages 378-397.

[10] Tobias R: Changing behavior by memory aids: a social psychological model of prospective memory and habit development tested with dynamic field data. Psychological Review, Vol 116(2), Apr 2009, 408-438. http://dx.doi.org/10.1037/a0015512

[11] Verplanken B, Wood W: Interventions to break and create consumer habits. Journal of Public Policy \& Marketing: Spring 2006, Vol. 25, No. 1, pp. 90-103. doi: http://dx.doi.org/10.1509/jppm.25.1.90

[12] Verplanken B, Melkevik O: Predicting habit: the case of physical exercise. Psychology of Sport and Exercise Volume 9, Issue 1, January 2008, Pages 15-26. 